\title{
Experiência Inicial no Fechamento Percutâneo da Comunicação Interatrial com a Prótese de Amplatzer
}

\author{
Valmir F. Fontes, Carlos A. C. Pedra, Simone R. F. Fontes Pedra, César A. Esteves, Sérgio L. N. Braga, \\ Jorge E. Assef, Sérgio C. Pontes Jr, Maria Virgínia T. Santana, Ziyad M. Hijazi, J. Eduardo M. R. Sousa
}

São Paulo, SP

\begin{abstract}
Objetivo - Analisar a experiência inicial no fechamento percutâneo da comunicação interatrial ostium secundum (CIA OS) com a prótese de Amplatzer.

Métodos - Sete pacientes foram submetidos ao procedimento através da via venosa anterógrada, orientados pela ecocardiografia transesofágica (ETE) e sob anestesia geral. Uma criança era portadora de 2 CIA e de canal arterial (CA). As CIA medidas pelo ETE variaram de 8,7 a 20mm. Um ecocardiograma transtorácico foi realizado na manhã seguinte do procedimento.

Resultados - Oito próteses foram implantadas nos 7 pacientes com sucesso. Em um paciente, o CA foi ocluído na mesma sessão com mola de Gianturco, tendo surgido taquicardia supraventricular durante a oclusão de uma das CIA, controlada com adenosina. Todos receberam alta hospitalar na manhã seguinte, com oclusão total dos defeitos.

Conclusão - O procedimento mostrou-se seguro, eficaz e versátil, podendo ser considerado como uma alternativa terapêutica inicial em pacientes selecionados com CIAOS.
\end{abstract}

Palavras-chave: comunicação interatrial, tratamento percutâneo, prótese de Amplatzer

\section{Initial Experience in Percutaneous Occlusion of Atrial Septal Defects with the Amplatzer Device}

Purpose - To evaluate our initial experience with percutaneous closure of secundum type atrial septal defects (ASD) with the Amplatzer septal occluder.

Methods - Seven patients underwent occlusion by anterograde approach, under general anesthesia and transesophageal echocardiography (TEE) guidance. One child had 2 ASD and a patent ductus arteriosus (PDA). The ASD size ranged from 8,7 to $20 \mathrm{~mm}$ as measured by TEE. A transthoracic echocardiogram was performed in the morning after the procedure.

Results - Eight devices were successfully implanted in 7 patients and the PDA was occluded with a Gianturco coil at the same session. In this patient, there was an episode of supraventricular tachycardia during the occlusion of one ASD which was reverted with adenosin. All patients were discharged the day after, with complete occlusion of all defects.

Conclusion - The procedure is safe, effective and versatile. It can be applied as an initial alternative to the treatment of selected patients with ASD.

Key-words: atrial septal defects, percutaneous treatment, Amplatzer device

Arq Bras Cardiol, volume 70 (n 3), 147-153, 1998

Desde a introdução da atrioseptostomia por cateter balão, idealizada por William Rashkind ${ }^{1}$, em 1966, muitas cardiopatias congênitas têm sido tratadas ou paliadas através do cateterismo intervencionista. A oclusão percutânea da comunicação interatrial (CIA) foi relatada, inicialmente, por King e Mills ${ }^{2}$, em 1974, porém, o sistema desenvolvido

Instituto Dante Pazzanese de Cardiologia

Correspondência: Valmir F. Fontes - Instituto Dante Pazzanese de Cardiologia, -

Av. Dr. Dante Pazzanese, 500 - 04012-180 - São Paulo, SP

Recebido para publicação em 27/11/97

Aceito em 5/1/98 não se popularizou, devido ao alto perfil dos introdutores necessários para o implante $(23 \mathrm{~F})$. Rashkind também criou um tipo de umbrella para o fechamento da CIA, que se fixava no septo através de minúsculos ganchos ou anzóis ${ }^{3}$. Essa particularidade dificultava o posicionamento adequado do dispositivo, além de predispor a possíveis complicações, sendo, portanto abandonado. No final da década de 80, Lock e col modificaram a umbrella dupla de Rashkind designada para o fechamento percutâneo do canal arterial, criando a clamshell double umbrella ${ }^{4}$. Esse dispositivo, após estudado extensivamente em modelos experimentais ${ }^{4}$, foi colocado na prática clínica com resultados promissores 
${ }^{5}$. No entanto, um grande estudo multicêntrico revelou que, no seguimento, algumas próteses apresentavam fraturas nas hastes de sustentação. Apesar deste achado não ter se associado a complicações clínicas, o FDA (Food and Drug Administration) suspendeu a utilização desta prótese até a resolução dos problemas técnicos. Todos esses trabalhos pioneiros deixaram bases sólidas para o desenvolvimento de novas técnicas de oclusão.

Nos dias de hoje, cinco próteses estão sendo avaliadas clinicamente, com resultados variáveis ${ }^{6-13}$. Neste artigo, relatamos a experiência inicial do Instituto Dante Pazzanese com a prótese de Amplatzer para a oclusão percutânea da CIA. Este dispositivo difere dos outros porque é baseado em um novo conceito para a oclusão: um núcleo metálico central (ou cintura), inserido no defeito como um stent, promovendo sua estabilidade. Os discos de cada lado da cintura amoldam-se ao lado esquerdo e direito do septo interatrial, suportando este núcleo central e otimizando seu poder oclusivo ${ }^{13}$.

\section{Métodos}

Pacientes com diagnóstico clínico (através de exame físico, radiografia de tórax, eletrocardiograma (ECG)) e ecocardiograma - pela ecocardiografia transtorácica - de CIA com repercussão hemodinâmica e indicação cirúrgica foram submetidos a estudo ecocardiográfico transesofágico para detalhamento anatômico do defeito. Foram considerados candidatos à oclusão percutânea os pacientes com os seguintes critérios de inclusão: 1) presença de CIA tipo ostium secundum; 2) diâmetro $<20 \mathrm{~mm}$; 3) dilatação do ventrículo direito (VD) com evidência de sobrecarga volumétrica; 4) shunt esquerdo-direito; 5) distância de pelo menos 4-5mm entre as margens do defeito e o seio coronário, valvas atrioventriculares e veia pulmonar superior direita e excluídos os casos de: 1) defeitos tipo ostium primum;2) defeitos tipo seio venoso; 3) drenagem anômala parcial ou total de veias pulmonares; 4 ) outros defeitos cardíacos associados que necessitassem de cirurgia cardíaca (por exemplo: comunicação interventricular); 5) hipertensão arterial pulmonar grave com shunt direito-esquerdo; 6) ou-

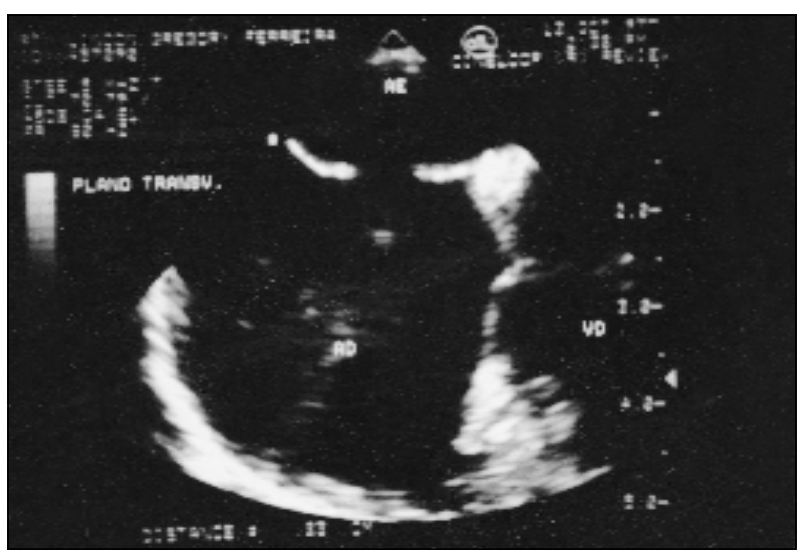

Fig. 1 - Ecocardiograma transesofágico do paciente 4 previamente ao implante - plano transverso (4 câmaras) mostrando CIA tipo ostium secundum de 10,3mm em seu maior diâmetro, distante das valvas atrioventriculares, veias pulmonares e seio coronário.

tros: trombose de cava inferior, sepsis, infecção pulmonar em atividade, doenças malignas com expectativa de vida $<3$ anos, presença de trombos intracardíacos, alergia a contraste iodados, crianças $<1$ ano.

Para os candidatos ao implante foi oferecida a opção do fechamento percutâneo com a prótese de Amplatzer. Os pacientes ou responsáveis foram orientados com relação a possíveis complicações do método, com esclarecimentos pertinentes e obtido consentimento por escrito para sua realização. O projeto de estudo também foi lido e aprovado pela comissão de ética da Instituição e pela Secretaria de Vigilância Sanitária do Ministério de Saúde do Brasil.

Sete pacientes foram submetidos ao fechamento percutâneo da CIA com a prótese de Amplatzer (tab. I). Todos apresentavam cansaço aos grandes esforços, não utilizavam medicação anticongestiva, e exibiam achados clássicos de CIA ao exame físico, morfologia de bloqueio de ramo direito ao ECG e aumento de trama vascular à radiografia de tórax. Área cardíaca aumentada foi constatada em cinco pacientes. Um apresentava sopro contínuo em região infraclavicular esquerda, detectando-se ao ecocardiograma transtorácico, um canal arterial de pequeno diâmetro.

As CIA foram minuciosamente avaliadas através do ecocardiograma transesofágico e medidas nos planos

\begin{tabular}{|c|c|c|c|c|c|c|c|}
\hline Paciente & 1 & 2 & 3 & 4 & 5 & 6 & 7 \\
\hline Idade (anos) & 5 & 13 & 10 & 7 & 5 & 9 & 12 \\
\hline Sexo & $\mathrm{F}$ & M & $\mathrm{F}$ & M & M & $\mathrm{F}$ & $\mathrm{F}$ \\
\hline Peso (kg) & 21 & 38 & 27 & 20 & 69 & 21 & 27 \\
\hline Clínica & $\begin{array}{c}\text { Cansaço } \\
\text { sinais de CIA }\end{array}$ & $\begin{array}{c}\text { Cansaço } \\
\text { sinais de CIA }\end{array}$ & $\begin{array}{c}\text { Cansaço } \\
\text { sinais de CIA }\end{array}$ & $\begin{array}{c}\text { Cansaço } \\
\text { sinais de CIA }\end{array}$ & $\begin{array}{c}\text { Cansaço } \\
\text { sinais de CIA }\end{array}$ & $\begin{array}{c}\text { Cansaço } \\
\text { sinais CIA/PCA }\end{array}$ & $\begin{array}{c}\text { Cansaço } \\
\text { sinais de CIA }\end{array}$ \\
\hline Radiografia de tórax pré & -TVB & $-A C,-T V B$ & -TVB & -AC, -TVB & -AC, -TVB & $-A C,-T V B$ & -AC, -TVB \\
\hline ECG & DCRD & DCRD & DCRD & DCRD & DCRD & DCRD & DCRD \\
\hline ETE CIA $(>$ Diam $)(\mathrm{mm})$ & 13,5 & 9,4 & 8,7 & 10,3 & 20 & $13 / 5$ & 16 \\
\hline DDFVD pré $\quad(\mathrm{mm})$ & 27 & 27 & 15 & 22 & 30 & 27 & 26 \\
\hline
\end{tabular}




\begin{tabular}{|c|c|c|c|c|c|c|c|}
\hline \multicolumn{8}{|c|}{ Tabela II - Dados durante e após o procedimento } \\
\hline Paciente & 1 & 2 & 3 & 4 & 5 & 6 & 7 \\
\hline $\mathrm{Qp} / \mathrm{Qs}$ & $>3$ & 1,6 & 1,5 & 2,4 & 2,6 & $>3$ & 1,6 \\
\hline \multicolumn{8}{|l|}{ Diâmetro estirado em (mm) } \\
\hline ETE & 17 & 14 & 12 & 12 & 23 & $21 / 9$ & 27 \\
\hline Prato & 18 & 15 & 13 & 13 & 24 & $21 / 9$ & 27 \\
\hline Prótese & 19 & $14 / 16$ & 13 & 13 & 24 & $22 / 8$ & 24 \\
\hline \multicolumn{8}{|l|}{ Shunt residual } \\
\hline Imediato & $\mathrm{N}$ & $\mathrm{N}$ & $\mathrm{N}$ & $\mathrm{T}$ & $\mathrm{N}$ & $\mathrm{N}$ & $\mathrm{T}$ \\
\hline $24 \mathrm{~h}$ & $\mathrm{~N}$ & $\mathrm{~N}$ & $\mathrm{~N}$ & $\mathrm{~N}$ & $\mathrm{~N}$ & $\mathrm{~N}$ & $\mathrm{~N}$ \\
\hline \multicolumn{8}{|l|}{ Tempo de } \\
\hline fluoroscopia (min) & 11,7 & 24,3 & 13,4 & 13,7 & 11 & 63,1 & 18,3 \\
\hline \multicolumn{8}{|l|}{ Tempo de } \\
\hline proc. $(\min )$ & 60 & 120 & 60 & 75 & 60 & 180 & 75 \\
\hline Radiografia de tórax pós & AC mant. & AC mant. & AC mant. & AC mant. & $\downarrow_{\mathrm{AC}}$ & $\downarrow_{\mathrm{AC}}$ & AC mant. \\
\hline $\begin{array}{l}\text { DDFVD pós } \\
(\mathrm{mm})\end{array}$ & 24 & 23 & 13,5 & 20 & 28 & 24 & 24 \\
\hline
\end{tabular}

transverso (4 câmaras) (fig. 1) e longitudinal (eixo das cavas). O maior diâmetro obtido em um destes planos foi tomado como o de referência, variando entre 8,7 a $20 \mathrm{~mm}$ (tab. I). No portador de canal arterial, evidenciou-se a presença de duas CIA, uma $>13 \mathrm{~mm}$ e outra $<5 \mathrm{~mm}$, distantes $8 \mathrm{~mm}$ uma da outra. Foram realizadas medidas do diâmetro diastólico final do VD ao modo M, antes e depois do procedimento. Todos os pacientes apresentavam aumentos variados das câmaras direitas traduzindo uma sobrecarga volumétrica.

A prótese de Amplatzer é constituída de dois discos auto expansíveis, conectados a um pequeno núcleo central (ou cintura), que corresponde ao diâmetro estirado da CIA. Todo o dispositivo é composto por uma rede metálica flexível de nitinol, preenchido internamente por remendos de poliéster para aumentar seu poder oclusivo (fig. 2). Estes remendos são costurados à rede metálica por monofi-

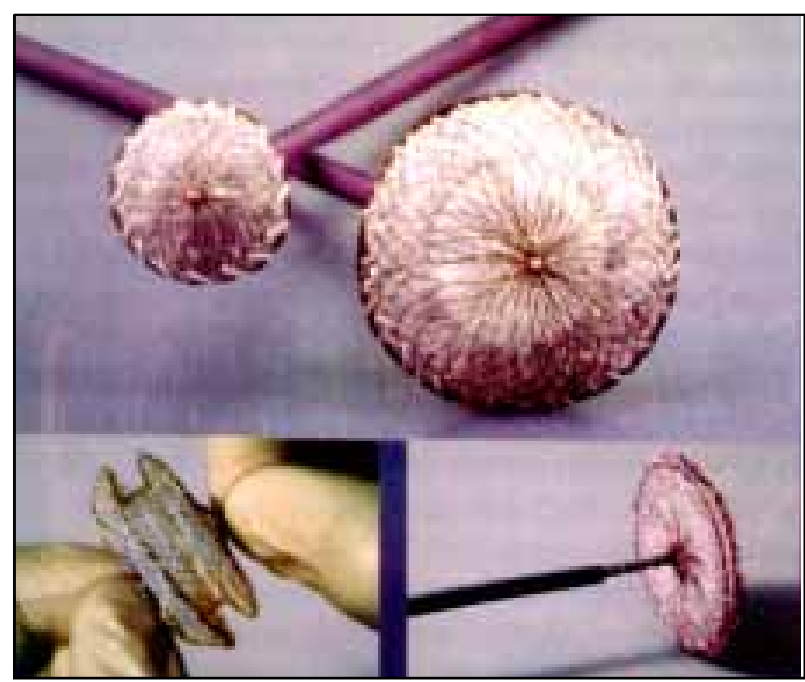

Fig. 2 - Prótese de Amplatzer - duplo disco de nitinol, auto-expansíveis, conectados por uma pequena cintura e preenchidos internamente por fibras de poliéster. A prótese conecta-se ao cabo liberador através de um mecanismo simples de rotação horária (parafuso). lamentos de poliéster. Os diâmetros disponíveis das próteses (correspondentes à cintura) vão de 4 a $26 \mathrm{~mm}$. Os discos são maiores que a cintura em 16 e $10 \mathrm{~mm}$, respectivamente, para o disco distal e proximal. As próteses até $10 \mathrm{~mm}$ são implantadas através de bainhas $6 \mathrm{~F}$ ou 7F, as de $10 \mathrm{a}$ $17 \mathrm{~mm}$ através de bainhas $7 \mathrm{~F}$ e as de 18 a $26 \mathrm{~mm}$ através de bainhas $8 \mathrm{~F}$.

Todos os pacientes foram submetidos à oclusão percutânea da CIA, orientada através da ecocardiografia transesofágica (ETE), sob anestesia geral balanceada, intubação orotraqueal e monitorização não invasiva anestésica. Foram puncionadas a veia e artéria femoral direitas e introduzidas bainhas $7 \mathrm{~F} \mathrm{e} 5 \mathrm{~F}$, respectivamente. Realizada heparinização na dose de 50U/kg e estudo hemodinâmico completo com coleta de amostras para oximetria. Todos os pacientes receberam antibioticoterapia profilática com cefazolina na dose de $30 \mathrm{mg} / \mathrm{kg} / \mathrm{dose}$, administradas antes do procedimento e de $6 / 6 \mathrm{~h}$ por mais três doses. Uma angiografia na veia pulmonar superior direita em projeção oblíqua esquerda $\left(30^{\circ}\right)$ com angulação cranial $\left(30^{\circ}\right)$ foi realizada para delineamento do(s) defeito(s) septal(is) (fig. 3). Um fio guia de troca 0.038 " foi deixado na veia pulmonar superior esquerda ou no átrio esquerdo (AE). Um cateter balão medidor (Med-tech 27/8/8/100) foi introduzido por sobre o guia e posicionado na porção livre do AE. O balão foi inflado com contraste diluído e puxado, cuidadosamente, até se ancorar no septo interatrial (fig. 4A e 4B). Após constatarmos, através da ecocardiografia, que o defeito estava ocluído totalmente pelo balão, foi gradualmente esvaziado até "pular" para o átrio direito (AD) com mínima resistência. Neste ponto, o diâmetro do balão foi medido pela ecocardiografia. $\mathrm{O}$ volume de contraste ainda presente no balão foi anotado, o balão foi retirado e reinflado fora do organismo com o mesmo volume de contraste. Este diâmetro foi comparado a diâmetros pré estabelecidos em uma placa medidora perfurada e foi definido como o diâmetro estirado da CIA. O procedimento de mensuração deste diâmetro foi 


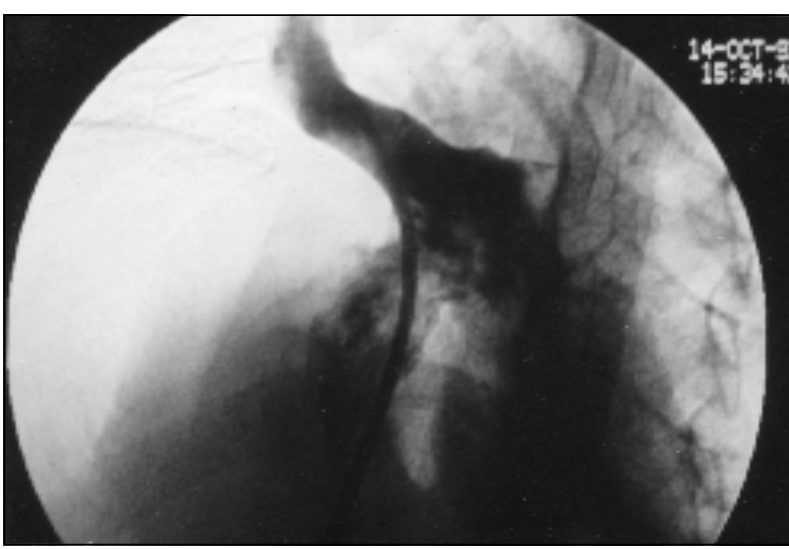

Fig. 3 - Angiografia na veia pulmonar superior direita do paciente 4 em projeção hepatoclavicular: CIA ampla localizada nas porções medianas do septo interatrial que se encontra bem delineado.

repetido por pelo menos mais uma vez. De modo geral, o dispositivo foi selecionado de forma que o diâmetro de seu núcleo central ou cintura fosse $\geq 1 \mathrm{~mm}$ que o diâmetro estirado da CIA. Uma bainha longa foi introduzida até o AE e posicionada em sua porção livre, distante do apêndice atrial
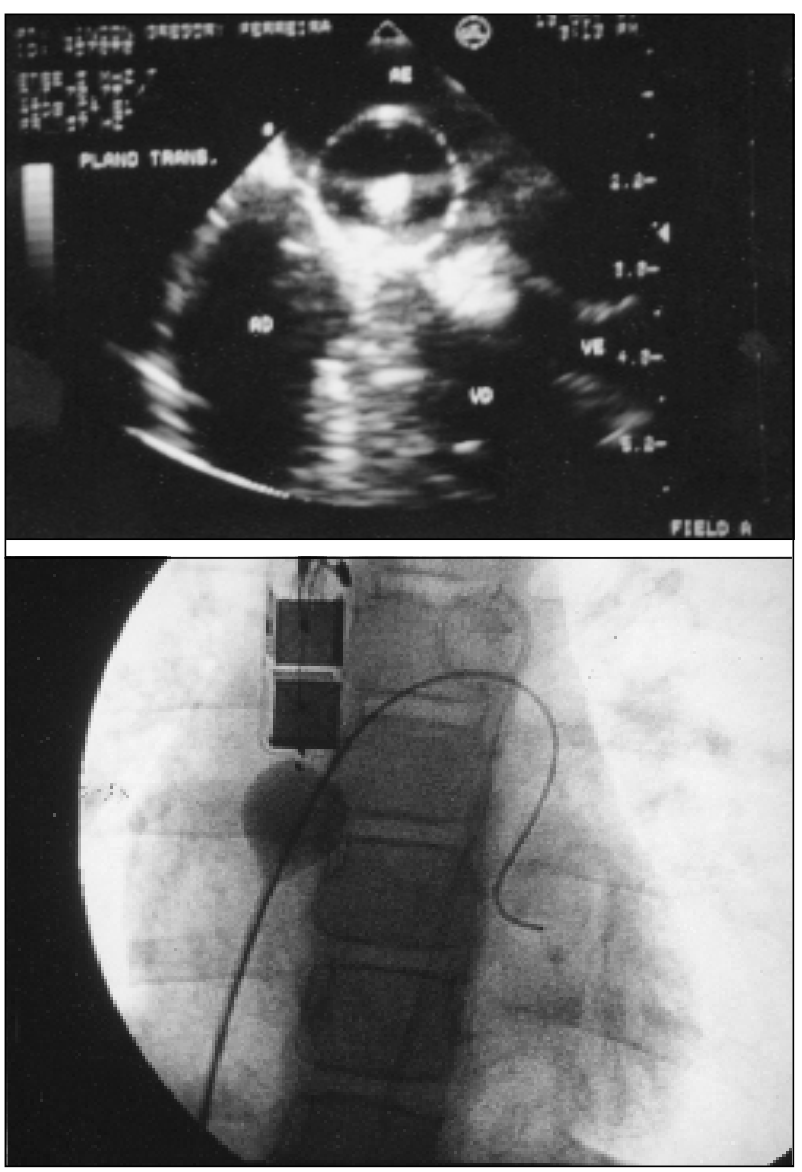

Fig. 4 - Mensuração de diâmetro estirado da CIA - nota-se balão inflado átrio esquerdo e tracionado de encontro ao septo interatrial: A) imagem ecocardiográfica no plano transverso; B) imagem obtida através de fluorocopia. Após redução progressiva do volume do balão, há súbita redução da resistência com passagem abrupta para o átrio direito. Neste ponto o diâmetro do balão é medido pela ecocardiografia. Após ter seu volume restaurado fora do organismo, o diâmetro é comparado a diâmetros pré estabelecidos em uma placa medidora.

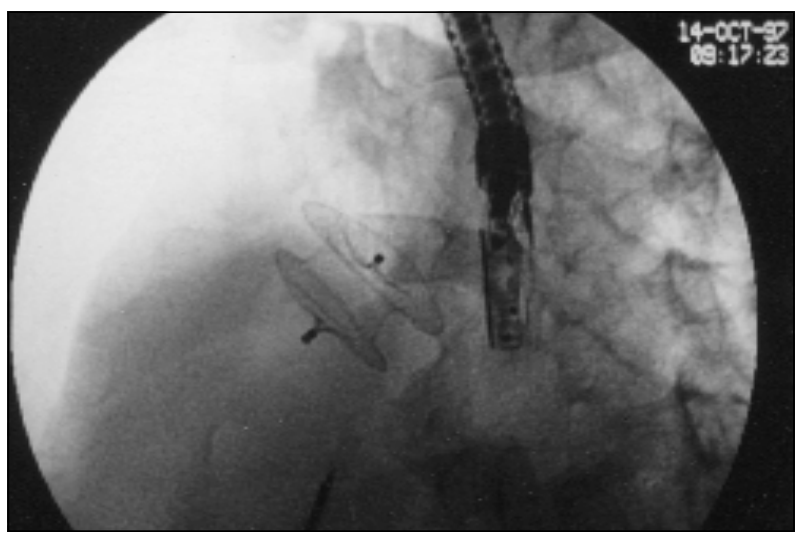

Fig. 5 - Prótese implantada - por ser um dispositivo auto centralizável, a prótese adquire um posicionamento ideal, paralela ao septo interatrial, após seu desacoplamento. Notem como a cintura fica inserida no defeito como um stent e como os discos se amoldam de cada lado do septo.

e da valva mitral. O dispositivo selecionado foi conectado a um cabo liberador através de um sistema de rotação horária (como um parafuso) e tracionado sob selo d'água para dentro de um carregador. $O$ carregador foi inserido firmemente dentro da bainha longa e o dispositivo foi avançado empurrando-se o cabo liberador. Esta manobra e todas as anteriores que requereram manipulação de cateteres no AE, só foram realizadas após certificação da ausência de bolhas de ar no sistema. Sob monitorização fluoroscópica, o dispositivo foi avançado até que o disco distal se abrisse no AE, distante do apêndice atrial e da valva mitral. Todo o sistema foi recuado até que o disco distal se ancorasse no lado esquerdo do septo interatrial. Mantendo-se um tração suave no cabo, a bainha foi retraída, progressivamente, até que o núcleo central se abrisse na CIA e o disco proximal no AD. A tração no cabo foi então aliviada para que o disco direito retomasse a sua configuração original, adaptando-se ao septo. $\mathrm{O}$ posicionamento adequado da prótese foi verificado, através da ecocardiografia e da manobra de Minnesota (Minnesota wiggle). Esta manobra é caracterizada por movimentos suaves que empurram e tracionam o dispositivo sem a capacidade de deslocá-lo do septo. Um adaptador de plástico foi conectado ao cabo liberador, que foi então girado no sentido anti-horário (movimento de desparafusar), liberando-se a prótese (fig. 5). O desacoplamento foi verificado pelo ecocardiograma e pela fluoroscopia. A presença de shunt residual imediata foi avaliada pelo mapeamento de fluxo a cores (fig. 6) e pela análise da recirculação após angiografia na artéria pulmonar em três pacientes.

No portador de canal arterial aliado a duas CIA, uma angiografia na aorta na projeção lateral esquerda foi realizada após os estudos pressóricos e oximétricos. O canal arterial mediu 2,2mm em seu menor diâmetro e foi ocluído com uma mola de Gianturco 38-8-5 (Cook Inc, Bloomington, IN) através da via arterial retrógrada, de acordo com técnicas já estabelecidas ${ }^{14}$. O procedimento de mensuração de cada CIA obedeceu os mesmos passos já descritos. Para o procedimento de oclusão propriamente dito, uma bainha longa foi posicionada através de cada CIA, sendo carregada com o dispositivo apropriado para cada orifíco. As próteses fo- 


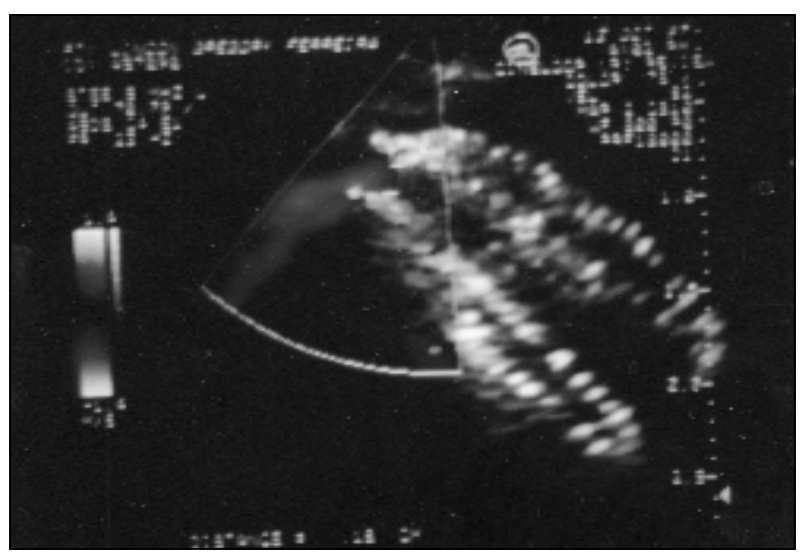

Fig. 6 - A presença de shunt residual é verificada ainda no laboratório de cateterismo através do ecocardiograma transesofágico com mapeamento de fluxo em cores. Neste caso houve oclusão imediata do defeito. Note-se como a prótese tem boa ecogenicidade permitindo fácil identificação e delineamento de seu posicionamento.

ram então implantadas, seqüencialmente, obedecendo o mesmo rigor técnico já apresentado. $\mathrm{O}$ tempo de procedimento e de fluoroscopia relativos a cada caso encontram-se na tabela II.

O sistema de implante da prótese de Amplatzer apresenta uma outra característica, que merece ser descrita. Se houver algum erro ou dúvida de posicionamento em qualquer um dos passos citados, o dispositivo pode ser facilmente resgatado para dentro da bainha, simplesmente, tracionando-se o cabo liberador. Esta capacidade permite reposicionar o dispositivo quantas vezes forem necessárias, até que haja certeza da sua localização, para só então proceder sua liberação final.

Após o implante os pacientes foram encaminhados a uma unidade de terapia intensiva para recuperação anestésica. Na manhã seguinte do procedimento, foram realizados ECG, radiografia de tórax e ecocardiograma transtorácico. Confirmada a estabilidade da prótese, os pacientes receberam alta hospitalar, orientados para receber ácido acetil salicílico (aproximadamente $5-10 \mathrm{mg} / \mathrm{kg} / \mathrm{dia}$ ) por seis meses e realizar profilaxia para endocardite infecciosa, quando necessária, pelo mesmo período de tempo. Tal conduta foi realizada a fim de prevenir a ocorrência de possíveis fenômenos tromboembólicos e/ou a instalação de endocardite até que se complete o processo de endotelização da prótese. Novos exames foram agendados com 1, 3 (aqui incluindo novo ETE) e 12 meses de seguimento.

\section{Resultados}

Os achados hemodinâmicos e anatômicos de cada caso encontram-se na tabela II. O diâmetro estirado da CIA estimado através do prato medidor guardou uma boa correlação com o mensurado através da ecocardiografia. Este diâmetro foi maior que o diâmetro real da CIA medido através da ETE, realizada antes do procedimento, variando entre 114 a $141 \%$. Em quatro pacientes, o implante foi realizado sem dificuldades. Os tempos de fluoroscopia e de procedi-
Fechamento percutâneo e CIA com prótese de Amplatzer

mento (pele a pele) foram extremamente satisfatórios em cinco pacientes (tab. II).

No paciente 2 houve uma dúvida em relação ao posicionamento final da prótese (de $14 \mathrm{~mm}$ ). Havia uma impressão que a parte inferior do disco proximal protruía-se para dentro do AE. A prótese foi então retirada e trocada por uma de $16 \mathrm{~mm}$. Depois do implante desta $2^{\mathrm{a}}$ prótese, certificamos que, na verdade, a porção inferior do septo interatrial é que era muito fina, levando à falsa impressão da protusão do dispositivo. Provavelmente, a prótese anterior estava em local adequado e poderia ter sido liberada sem intercorrências.

No paciente 6, após a oclusão do canal arterial, optamos por abordar inicialmente a CIA de maior diâmetro, localizada em porções superiores do septo. Quando o disco distal de uma prótese 22 começou a ser aberto no AE, o paciente apresentou episódio de taquicardia supraventricular (TSV) revertida prontamente com a administração de adenosina. Verificou-se que não havia espaço suficiente para a completa expansão do disco, devido à presença da outra bainha posicionada no AE e que passava através da CIA inferior. A prótese 22 foi recolhida para dentro da bainha e a estratégia do procedimento foi modificada. A CIA de menor diâmetro foi então abordada, primeiramente, e uma prótese de $8 \mathrm{~mm}$ foi, adequadamente, posicionada sem intercorrências, criando-se assim, mais espaço no AE e a prótese de $22 \mathrm{~mm}$ pôde ser aberta e posicionada corretamente na maior CIA, sem novos episódios de TSV. Após confirmação das suas estabilidades, ambos dispositivos foram seqüencialmente desconectados do cabo liberador sem intercorrências. No final, as porções inferiores de ambos os discos da prótese superior cobriram as porções superiores da prótese inferior, lembrando imagem de sanduíche.

No paciente 7, apesar do diâmetro real da CIA ter medido $16 \mathrm{~mm}$, o diâmetro estirado, estimado várias vezes através do prato medidor e da ecocardiografia, foi de $27 \mathrm{~mm}$. Constatamos que as bordas da CIA apresentavam septo de espessura muito delgada, que não opunham resistência à tração suave do cateter balão, explicando a discrepância. Arbitrariamente, selecionamos uma prótese de $24 \mathrm{~mm}$ para o implante. $\mathrm{O}$ dispositivo só foi, adequadamente, posicionado na $4^{\mathrm{a}}$ tentativa, já que por duas vezes o disco distal não se ancorou no lado esquerdo do septo, "pulando" para o AD e, por uma vez, ambos os discos foram equivocadamente abertos no AE.

Com exceção do paciente que apresentou TSV paroxística, não tivemos complicações. Não ocorreu nenhum episódio de embolização ou de derrame pericárdico. Cinco pacientes deixaram o laboratório de cateterismo sem shunt residual e à alta todos apresentavam oclusão completa dos defeitos, incluindo a portadora de canal arterial. Em nenhum paciente constatou-se disfunção das valvas AV ou distúrbios de fluxo nas veias cavas, seio coronário e veias pulmonares. Todos os pacientes apresentaram discreta redução do diâmetro diastólico final do VD na manhã seguinte do procedimento. Todos deixaram o hospital em ritmo sinusal e, em dois de cinco pacientes, houve redução significativa da área cardíaca (tab. II). 


\section{Discussão}

ACIA é uma cardiopatia caracterizada pela deficiência estrutural do septo interatrial. Corresponde, aproximadamente, a 10-15\% de todas as cardiopatias congênitas e tem uma predominância no sexo feminino (3:2). O tipo mais freqüente é o defeito localizado na fossa oval (ostium secundum $)^{15}$. As conseqüências desta lesão dependem, basicamente, da magnitude e da duração do shunt, e do comportamento individualizado do leito vascular pulmonar. Em defeitos com grande shunt esquerdo direito, há uma sobrecarga volumétrica das câmaras direitas e desenvolvimento de hipertensão arterial pulmonar a longo prazo (habitualmente após a $3^{\circ}$ década de vida). Após a $4^{\circ}$ década, a frequiência de arritmias atriais também é maior nesses pacientes ${ }^{15}$. Por isto recomenda-se que pacientes com fluxo pulmonar $\geq 1,5$ vezes o fluxo sistêmico sejam operados por volta dos cinco anos de idade ou na vigência do diagnóstico após esta idade ${ }^{16}$.

A abordagem cirúrgica é o método convencional de tratamento da lesão, necessitando de um curto período de circulação extracorpórea. Apesar da cirurgia ser um procedimento seguro com índices de mortalidade de 1-3\% ${ }^{16}$, a ocorrência de shunts residuais parece ser mais comum do que se pensava anteriormente ${ }^{17}$. Infelizmente, nenhum estudo tem avaliado, sistematicamente, os resultados cirúrgicos através da color Doppler ecocardiografia, preferencialmente através da abordagem transesofágica. Com isto, shunts pequenos ou triviais podem passar desapercebidos clinicamente ou mesmo no laboratório de cateterismo ${ }^{18}$. De modo geral, estima-se que $2 \%$ dos pacientes são reoperados para tratamento de shunts residuais significativos ${ }^{16}$.

Desde o final da década passada, houve um ressurgimento no interesse do fechamento percutâneo da CIA. Vários dispositivos foram estudados com resultados satisfatórios, cada um apresentando características próprias e limitações ${ }^{6-13}$. Neste estudo inicial, demonstramos que a oclusão da CIA através da prótese de Amplatzer pode ser realizada com segurança e alta eficácia. Como é resgatável e reposicionável antes de sua liberação final, foi possível estender seu uso para casos de maior complexidade técnica, como ficou exemplificado nos casos 2,6 e 7. Esta característica não é observada em outros dispositivos.

Outra diferença em relação a outras próteses é a presença de um núcleo central ou cintura. Este núcleo fica inserido no defeito como um stent dando estabilidade ao dispositivo, dispensando a necessidade de braços ou esqueletos metálicos para tal finalidade. As fraturas que às vezes ocorrem nestas hastes, ficam assim evitadas. Além disto, esta cintura centraliza, automaticamente, a prótese em relação ao septo independente do ângulo de ataque para o implante, facilitando seu posicionamento. Como os discos também não precisam apresentar grandes dimensões para estabilização do dispositivo, seu implante pode ser potencialmente estendido a defeitos que não apresentam bordas extensas que o separem de outras estruturas intracardíacas. Este aspecto torna-se relevante, já que na prática clínica, temos visto vários casos de CIA tipo ostium secundum com deficiência da porção anterior do septo. Na verdade, a principal função dos discos é de otimizar o poder oclusivo da prótese devido à presença de fibras de poliéster em seu interior que contribuem para obstruir o fluxo transeptal. Estes discos, assim como toda a estrutura metálica de nitinol, são extremamente flexíveis e não contêm elementos ponteagudos ou ganchos, minimizando o risco de perfurações durante ou após o procedimento.

A técnica de implante é simples, permitindo rápida familiarização a operadores com experiência em cardiologia intervencionista pediátrica. Considerando que esta casuística representa parte da nossa curva de aprendizado inicial, os procedimentos foram realizados em pouco tempo e com uso criterioso da fluoroscopia. As bainhas necessárias para o implante são de baixo perfil, possibilitando a extensão da técnica a crianças de baixo peso e/ou menores que um ano de idade.

Como já foi comentado, a cintura exerce um papel fundamental para estabilidade e poder oclusivo desta prótese. Por isto, seu diâmetro necessita ter dimensões muito próximas do diâmetro estirado da CIA, sendo, portanto, de vital importância que a mensuração deste diâmetro seja realizada de forma precisa, devendo ser repetida quantas vezes forem necessárias. Este passo pode levar mais tempo que o implante em si, explicado talvez pela ausência de cateteres balão, especificamente, designados para tal finalidade ${ }^{13}$.

Nossos resultados iniciais de $100 \%$ de oclusão são compatíveis com os resultados do estudo multicêntrico internacional, realizado para avaliação clínica desta prótese. Em mais de 400 casos realizados, o índice de oclusão chegou a 95\% após três meses de seguimento, caracterizando sua alta eficácia (Ziyad M. Hijazi, comunicação pessoal).Éinteressante notar como houve discreta redução das dimensões do VD e da área cardíaca em dois pacientes, já no dia seguinte após a oclusão. A quase totalidade dos pacientes que não apresenta oclusão completa, permanece com shunts residuais triviais a pequenos (segundo a classificação de Toronto ${ }^{19}$ ), sem repercussão hemodinâmica, podendo ser considerados como clinicamente tratados. No entanto, como não conhecemos a história natural desses pacientes, tornam-se candidatos a receber profilaxia para endocardite infecciosa por toda a vida. Como em qualquer procedimento intervencionista, o implante da prótese de Amplatzer também pode se associar a complicações. Em nossa casuística, a ocorrência da TSV observada no paciente 6 esteve mais relacionada a uma tática inicial inadequada para a abordagem das duas CIA, do que ao implante propriamente dito. No estudo multicêntrico, complicações menores como extrasístoles atriais sem repercussão hemodinâmica puderam ser observadas após a abertura do disco distal. Como complicações maiores foram notados dois casos de embolização do dispositivo, um resgatado do VD, ainda no próprio laboratório de cateterismo com implante de um $2^{\circ}$ dispositivo em uma outra oportunidade, e outro, resgatado da artéria pulmonar na sala cirúrgica, com fechamento da CIA. Nenhum 
desses pacientes apresentou repercussões clínicas deletérias secundárias à embolização. Em dois pacientes foram verificados episódios de ataque isquêmico transitório, provavelmente, relacionados à liberação de microcoágulos do disco distal. Ambos evoluíram sem seqüelas clínicas e um necessitou da retirada cirúrgica do dispositivo. Um lactente apresentou quadro séptico não relacionado ao implante três meses após o procedimento, evoluindo com endocardite no local da prótese, necessitando da sua retirada cirúrgica. Estes episódios refletem a importância da orientação para antiagregação plaquetária e para profilaxia da endocardite. Até agora não se observou nenhum caso de óbito relacionado ao procedimento (Ziyad M. Hijazi, comunicação pessoal). Este baixo índice de complicações denota o grau de segurança do método.

O implante de dois dispositivos para a oclusão de duas CIA ainda não é relatado na literatura internacional, apesar de já ter sido realizado (Ziyad M. Hijazi, comunicação pessoal). Este tipo de procedimento associado ao fechamento percutâneo do canal arterial, como foi observado no paciente 6 , é inédito e merece destaque.

É importante ressaltar o papel da ecocardiografia no implante. Muito do sucesso alcançado nesta experiência pode ser atribuído ao rigor técnico que esses pacientes foram previamente selecionados. A caracterização anatômica detalhada do tipo e da localização da CIA no septo, do número de orifícios, da distância do defeito em relação a estruturas intracardíacas e da presença de outros defeitos associados são parâmetros indispensáveis para o implante ${ }^{20}$. Ao nosso ver, estes dados são obtidos de forma mais fidedigna através da Doppler ETE com mapeamento de fluxo a cores. Durante o procedimento, este método assume fundamental importância na mensuração do diâmetro estirado da CIA, na avaliação do posicionamento final da prótese e da presença de shunt residual imediato.

Concluindo, o fechamento percutâneo da CIA através da prótese de Amplatzer é um procedimento relativamente simples, com resultados iniciais encorajadores e que devido a sua versatilidade, pôde ser estendido para casos de maior complexidade anatômica, como a oclusão de duas CIA ou de defeitos com bordas delgadas. Dada as óbvias vantagens em relação ao tratamento cirúrgico convencional (conforto ao paciente, ausência de cicatriz cirúrgica, tempo de internação e custo, entre outras), é provável que este método se torne de primeira linha na abordagem terapêutica da maior parte das CIA localizada na fossa oval.

\section{Agradecimentos}

À Fundação Adib Jatene pelo auxílio financeiro recebido.

\section{Referências}

1. Rashkind WJ, Miller WW - Creation of an atrial septal defect without thoracotomy: a palliative approach to complete transposition of the great vessels. J Am Med Ass 1966; 196: 991-2.

2. King TD, Mills NL - Secundum atrial septal defects: non operative closure during cardiac catheterization. J Am Med Ass 1976; 235: 2506-9.

3. Rashkind WJ - Transcatheter treatment of congenital heart disease. Circulation 1983; 67: 711-16.

4. LockJE, Rome JJ, Davis Ret al - Transcatheter closure of atrial septal defects: Experimental studies. Circulation 1989; 79: 1091-9.

5. Rome JJ, Keane JF, Perry SB, Spevak PJ, Lock JE - Double umbrella closure of atrial septal defects. Initial clinical aplications. Circulation 1990; 82: 751-8.

6. Rao PS, Sideris EB, Hausdorf G et al - International experience with secundum atrial septal defect occlusion by the buttoned device. Am Heart J 1994; 128: 1022-35.

7. Haddad J, Secches A, Finzi L et al - Oclusão percutânea transvenosa da comunicação interatrial mediante a utilização do buttoned device. Arq Bras Cardiol 1996; 67: 17-22.

8. Das GS, Voss G, Jarvis G, Wyche K, Gunther R, Wilson RF - Experimental atrial septal defect closure with a new transcatheter, self centering device. Circulation 1993; 88(part 1): 1754-64.

9. Sievert H, Babic UU, Ensslen R et a -. Vershulss des vorhofseptumdefektes mit einem neuen Okklisionssystem. Z Kardiol 1996; 85: 97-103.

10. CardioSeal septal occlusion system. Nitinol Medical Technologies, Inc. USA Brochure, 1997.

11. Sharafundin MJA, Gu X, Titus J et al - Transvenous closure of secundum atrial septal defects: preliminary results with a new self expanding nitinol prosthesis in a swine model. Circulation 1997; 95: 2162-8.
12. Bjornstad PG, Smevik P, Fiane AE et al - Catheter-based closure of atrial septal defects with a newly developed nitinol double disc: an experimental study. Cardiol Young 1997; 7: 220-4.

13. Bjornstad PG, Masura J, Thaulow E et al - Interventional closure of atrial septal defects with the Amplatzer device first clinical experience. Cardiol Young 1997; 7: 277-83.

14. Pedra CAC, Esteves CA, Braga SLN, Kambara A, Fontes VF - Oclusão percutânea do canal arterial: estado da arte. Rev Bras Cardiol Inv 1997; 5: 22-35.

15. Medeiros Sobrinho JH, Fontes VF, Pontes Jr SC - Defeitos isolados do septo atrial. In: Medeiros Sobrinho JH, Fontes VF, Pontes Jr SC, eds - Cardiopatias Congênitas. São Paulo: Sarvier, 1990: 295-314.

16. Kirklin JW, Barrat Boyes G - Atrial septal defects and partial anomalous pulmonary venous connections. In: Kirklin JW, Barrat Boyes G, eds - Cardiac Surgery. $2^{\text {nd }}$ ed. New York :Churchill Livingstone, 1993: 609-44.

17. Santoso T, Meltzer RS, Castellanos S, Serruys PW, Roelandt J - Contrast echocardiographic shunts may persist after atrial septal defect repair. Eur Heart J 1983; 4: 129-33.

18. Grossman W-Shunt detection and measurement. In: Baim DS, Grossman W, eds - Cardiac Catheterization, Angiography and Intervention. $5^{\text {th }}$ ed.. Baltimore: William \& Wilkins, 1996: 167-82.

19. Boutin C, Musewe NN, Smallhorn JF et al - Echocardiographic follow-up of atrial septal defect after catheter closure by double umbrella device. Circulation 1993; 88: 621-7.

20. Magni G, Hijazi ZM, Pandian NG et al - Two and three dimensional transesophageal echocardiography in patient selection and assessment of atrial septal defect closure by the new DAS-Angel Wings device: initial clinical experience. Circulation 1997; 96: 1722-8. 